\title{
Simulations on Consumer Tests: A Systematic Evaluation Approach in an Industrial Case Study
}

\author{
Christian Berger*, Delf Block ${ }^{\ddagger}$, Sönke Heeren ${ }^{\dagger}$, Christian Hons ${ }^{\ddagger}$ \\ Stefan Kühnel ${ }^{\ddagger}$, André Leschke ${ }^{\ddagger}$, Dimitri Plotnikov ${ }^{\S}$, and Bernhard Rumpe ${ }^{\S}$ \\ ${ }^{*}$ Department of Computer Science and Engineering, Chalmers - University of Gothenburg, Sweden \\ ${ }_{\ddagger}^{\ddagger}$ Volkswagen AG, Development Car Safety, Electronic and Testing — Wolfsburg, Germany \\ ${ }^{\dagger}$ Automotive Safety Technologies GmbH, Competence Cluster Wolfsburg — Ingolstadt, Germany \\ $\S$ Software Engineering, RWTH Aachen, Germany
}

\begin{abstract}
Context: Consumer tests for vehicles have long a tradition of almost two decades in Europe to assess vehicular safety abilities. For active safety systems like an emergency braking guard, different consumer-test-organizations (CTOs) around the world intensify now the challenges for automotive Original Equipment Manufacturer (OEM) in terms of specific test protocols.

Objective: This work focuses on the systematic generation of possible vehicle trajectories within the allowed tolerance ranges for a simulation environment. It is of great interest to which extent the different test parameters will affect the brake timepoints in each test case as well as the residual velocity in case of a collision with a target vehicle.

Method: A formal model using a graph-based representation of the allowed variances based on relevant time-points allows the selection of relevant trajectories as test cases. These trajectories are simulated afterwards to investigate its influence on the system's performance.

Results: The systematic approach unveiled varying action points in time of the software of an emergency braking guard, which could influence the overall result of a consumer test.

Conclusion: The use of a structured simulative approach for evaluating an active safety system during the development process enables a more focused feedback and supports extensive tests on real proving grounds by systematic and automated robustness analyses for example.
\end{abstract}

\section{INTRODUCTION AND MOTIVATION}

Advanced Driver Assistance System (ADAS) are playing an increasing role as safety features even in the lower classes of today's vehicle sizes like Golf, Polo, or up!. The development of active safety systems is a challenging task because such systems are designed to operate in potentially dangerous crash- and near-crash-scenarios. Furthermore, detecting such hazardous situations and reacting as quick as possible is of central interest and will probably result in a better rating at CTOs. Additionally, consumers have their individual driving styles, so specific requirements have to be fulfilled to match their expectations regarding the system's behavior without infantilizing them in their driving style. Reaching these two development goals by finding a robust parameters set for the active safety system to meet the consumer's expectation is distinctive for the development of today's automotive safety functions.

\section{A. Problem Domain and Motivation}

As from 2014, European New Car Assessment Programme (EuroNCAP) started assessing active safety systems like Forward Collision Warning (FCW) and Autonomous Emergency Braking (AEB) besides others. Additionally, consumers as well as OEMs are interested in receiving the top rating for the safety features of their cars. EuroNCAP specified these testing procedures and the allowed variation of several test parameters within certain tolerance ranges in detail [1].

As shown in our previous work [2], these ranges influence the assessment and could result in an unwanted minor rating of the active safety system and the vehicle itself. Therefore, real test runs are inevitable to evaluate the performance of such systems; however, a simulation approach will provide additional insights and allows further analysis of the system's behavior that are hardly achievable in reality due to riskiness of the test scenarios or controllability of the boundary conditions.

\section{B. Research Goal and Research Questions}

The research goal for this study is to systematically evaluate the EuroNCAP assessment procedure on the example of the "Car-To-Car-Rear: stationary (CCRs)" test in a simulationbased environment for analyzing the impact of parameter variations within the allowed tolerance ranges. Thus, the following research questions are of specific interest:

RQ-1: How can specific parameters within particular tolerance ranges be modeled and varied to simulate different trajectories of the Vehicle-UnderTest (VUT) driving towards a target vehicle in a systematic way?

RQ-2: To which extent would different lateral positions and heading of the vehicle outside the allowed tolerance ranges by EuroNCAP influence an AEB/FCW algorithm and further the residual velocity in case of a collision between VUT and the target vehicle?

In our case study, we focused on the CCRs scenarios for addressing the research questions and to have the basis for further studies. 


\section{Contributions of the Article}

In our work, we present a simulation approach that utilizes a graph-based model for EuroNCAP tests. By our experiments we could unveil the effect of lateral deviation on the trigger points of an emergency braking guard. Moreover, we show the maximum deviation from the ideal trajectory where no activation of the system will occur.

By using such an simulation environment, the engineers could be supported in efficiently planning real world experiments and concentrating on those test cases where anomalies have been detected.

\section{Structure of the Article}

In Sec. II, a selection of related work is presented; Sec. III illustrates the main boundary conditions of the EuroNCAP's test protocol regarding $\mathrm{AEB} / \mathrm{FCW}$ systems followed by a summary of the used simulation environment. Afterwards, the experiment for the CCRs test cases and its results are discussed in Sec. IV. The work is summarized and concluded in Sec. V.

\section{RELATED WORK}

Belbachir et al. present a method for assessing ADAS including an assessment architecture containing environmental and vehicle components in a simulation. The objective of this simulation-driven approach is to validate such systems by explicitly considering different self-designed evaluation criteria like pedestrian detection error or driver safety estimation [3].

The work of Rauskolb et al. describes the realization of an autonomous driving vehicle for the 2007 DARPA Urban Challenge. One part focuses especially on the use of a simulation environment for acceptance testing in accordance to the requirements given by that competition. This hardwareindependent approach aims on modifying object data within the restricted operating environment of the vehicle [4], [5].

Another hardware-independent simulation approach of ADAS is illustrated by Martinus et al. who developed a Virtual Application Platform (VAP) for Software-in-the-Loop (SiL)-tests to support the frontloading during the software development process of ADAS concentrating on functional tests. The virtual platform is based on the AUTOSARStandard to deploy software releases without the need for real hardware. Moreover, they also combined the VAP with virtual test driving including a vehicle dynamics model and the according environmental simulation without a special focus on the test scenarios [6].

The simulation approach by Nentwig et al. focuses using the original hardware of the supplier realizing a Hardware-inthe-Loop (HiL)-testbed based on the software tools Virtual Test Drive (VTD) and Automotive Data and Time Triggered Framework (ADTF). The simulation environment addresses the functional testing and system testing of video-based systems [7], [8].

Schick et al. worked on a similar research simulation framework for video-based ADAS. They use a different toolset provided by IPG Automotive $\mathrm{GmbH}$ in contrast to the aforementioned toolchain to access time-dependent data from virtual camera and radar sensors to validate sensor data fusion algorithms [9]. In [10], a use-case for evaluating a chassis control system is illustrated using the vehicle dynamics simulation of IPG.

Chucholowski et al. worked on a real-time numerical simulation environment to model the vehicle dynamics of a passenger car for the ISO slalom test [11]. Tideman et al. present the toolset "PreScan" by TNO on the basis of manually creating test scenarios and the evaluation of a Lane-Keeping Assist (LKA) from a functional point of view [12], [13].

To the best knowledge of the authors, the design of a structured experiment and its results from systematically applying a simulation-based approach to evaluate an active safety system according to a new car assessment program in a real industrial setting have not been published so far.

\section{SIMULATING CONSUMER TESTS}

At first, we are shortly presenting the purpose and structure of the EuroNCAP AEB test protocol for evaluating active safety systems for modern cars. Additionally, a key aspect of designing a simulation environment is briefly outlined.

\section{A. EuroNCAP's AEB Test Protocol}

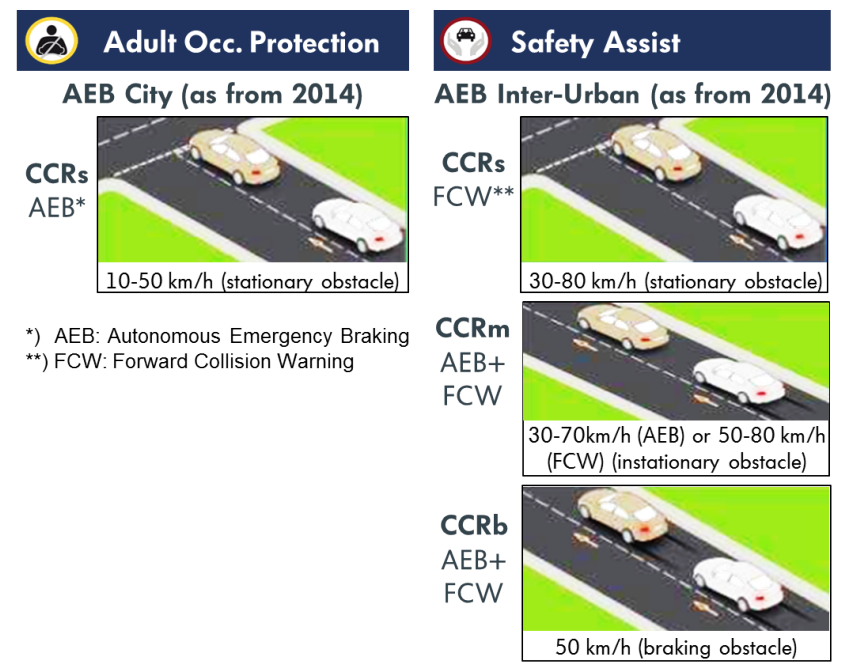

Fig. 1. Summary of EuroNCAP's test scenarios and the assessed functions (based on [14]).

EuroNCAP is a non-profit organization composed of several stakeholders including seven European Governments, motoring and consumer organizations. For a detailed description of the different tests and score calculation, we refer to [15].

Since 2014, there are three different test scenarios representing typical types of crashes occurring in city and inter-urban areas and being addressed by AEB/FCW systems as depicted by Fig. 1:

- Car-To-Car-Rear: stationary (CCRs)

- Car-To-Car-Rear: moving (CCRm)

- Car-To-Car-Rear: braking (CCRb) 
The description of the CCRs scenario is provided in Sec. IV. $\mathrm{CCRm}$ and $\mathrm{CCRb}$ are characterized by a moving target vehicle, driving at a speed of either $20 \mathrm{~km} / \mathrm{h}$ or $50 \mathrm{~km} / \mathrm{h}$ respectively, while the VUT's velocity ranges from 30 to 80 $\mathrm{km} / \mathrm{h}$ increased by $5 \mathrm{~km} / \mathrm{h}$ and depending on the assessed function. In 2016, additional test scenarios including different types of pedestrians will complement this catalogue.

For conducting a successful test on a real proving ground, several test parameters regarding the CCRs scenario have to be within the following ranges [1]:

- speed of VUT (test speed $+1.0 \mathrm{~km} / \mathrm{h}$ )

- lateral deviation from test path $(0 \pm 0.1 \mathrm{~m})$

- yaw velocity $(0 \pm 1.0 \% \mathrm{~s})$

- steering wheel velocity $(0 \pm 15.0 \%$ s $)$

These parameters are relevant between $4 \mathrm{~s}$ before the VUT probably hits the target vehicle depending on its test speed and the actual activation of the active safety system. Otherwise, the test is considered incorrect according to EuroNCAP's test protocol.

\section{B. Simulation-based Evaluation for Active Safety Systems}

There are different reasons why an automotive OEM is interested in simulating active safety systems and attaining additional insights with respect to real test runs on proving grounds. Firstly, the effort is quite high to prepare VUTs for such test runs with driving robots and adjusting their parameters for example. Furthermore, the ability to reproduce test runs is one of the major advantages of a simulation environment allowing a more systematic investigation of active safety systems than in reality. Nevertheless, simulated test runs will not replace runs on proving grounds like it is still common practice in developing passive safety features.

In [2] we outlined a method for designing a simulation environment more systematically. One key aspect in that work was to analyze the later scope of application for that environment. Two questions are fundamental for an engineer:

1) Which engineering problem should be addressed by the simulation?

2) Which result is finally desired?

The first question focuses on the concrete development task, for example "tolerance analysis wrt. to specific consumer test scenarios". The other question concentrates on defining the insight that should be attained by the simulation process because the model abstraction depends foremost on the desired result, in this case "Which parameter has a greater influence on the distribution of the remaining speed than others?" for example. The following section describes a technical solution to answer such a question.

\section{Simulation Runtime Environment}

The simulation environment consists of several software components whose elements are briefly described as they constitute the basis for our experiment in the industrial setting.
1) Virtual Test Drive: VTD is a software tool developed by the VIRES Simulationstechnologie $\mathrm{GmbH}$ for openSuSE Linux. It simulates automotive vehicles in a 3D virtual environment including surrounding objects like other traffic participants, pedestrians, vegetation, buildings, traffic signs, and terrain. It has a modular layout so that different vehicle dynamics and environmental sensor modules can be deployed for example. The individual modules communicate via the Runtime Data Bus (RDB), which provides detailed information about all objects; the Simulation Control Protocol (SCP) is used to control the simulation flow [16], [17].

2) Automotive Data and Time Triggered Framework: The ADTF is a software tool developed by the Audi Electronics Venture GmbH (AEV) either running on Windows or Linux platforms. It is used for the development and testing of driver assistance systems and safety functions in the automotive industry especially designed for recording and re-playing large amounts of vehicle data [18], [19].

3) Complementing Core Modules: The simulation runtime environment for controlled simulating of EuroNCAP test cases consists of two virtual machines running in an Oracle VirtualBox [20]. The first virtual machine is a Windows instance hosting the ADTF configuration including the emergency braking guard. The second virtual machine is a Linux openSuSE instance hosting the VTD environment.

The developed simulation architecture is comprised of a virtual driver module and a vehicle dynamics module. The former is implemented as an ADTF filter that provides stimuli data like braking and acceleration rates for the emergency braking guard running in the ADTF configuration; the latter positions the VUT on the precomputed coordinates in the VTD scene during a simulation run.

\section{SYSTEMATIC EVALUATION OF TEST CASE VARIATIONS FOR CCRS TESTS - AN INDUSTRIAL CASE STUDY}

In the following, we are describing our experimental study on systematically evaluating tolerance ranges for EuroNCAP test cases on the example of the CCRs test scenario in an industrial case study. We are reporting according to the guidelines from Jedlitschka et al. [21] and Runeson and Höst [22].

\section{A. Experimental Setup}

For our experimental setting, we are focusing on the CCRs scenarios that are characterized by a target vehicle as a static obstacle being placed in front of the VUT in a certain distance. Its velocity will be steadily increased by $5 \mathrm{~km} / \mathrm{h}$ steps from the interval $[10,50] \mathrm{km} / \mathrm{h}$ in each test case resulting in nine basic test cases. The VUT, which is equipped with an emergency braking system, has to drive with the constant test speed towards the target until the system performs the emergency braking maneuver.

For each test case there are specific points in time which are indicating the beginning of the test, its ending, and the actual activation of the active safety system. The test formally starts when the Time-To-Collision (TTC) equals $4 \mathrm{~s}\left(T_{0}\right)$ and 


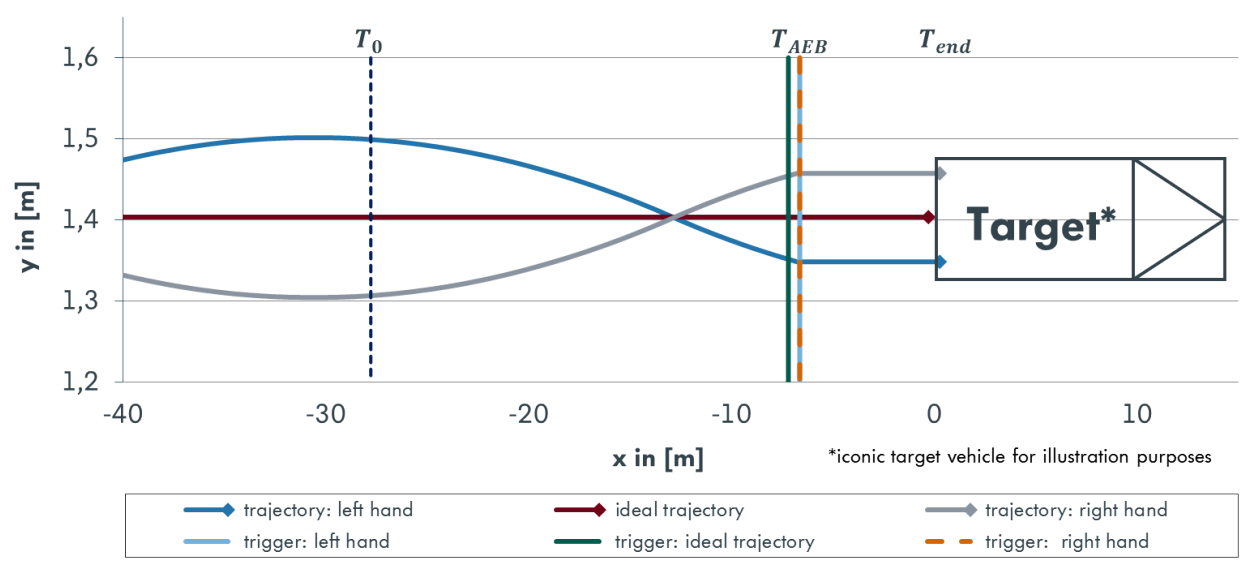

Fig. 3. CCRs test case with a test speed of $25 \mathrm{~km} / \mathrm{h}$.

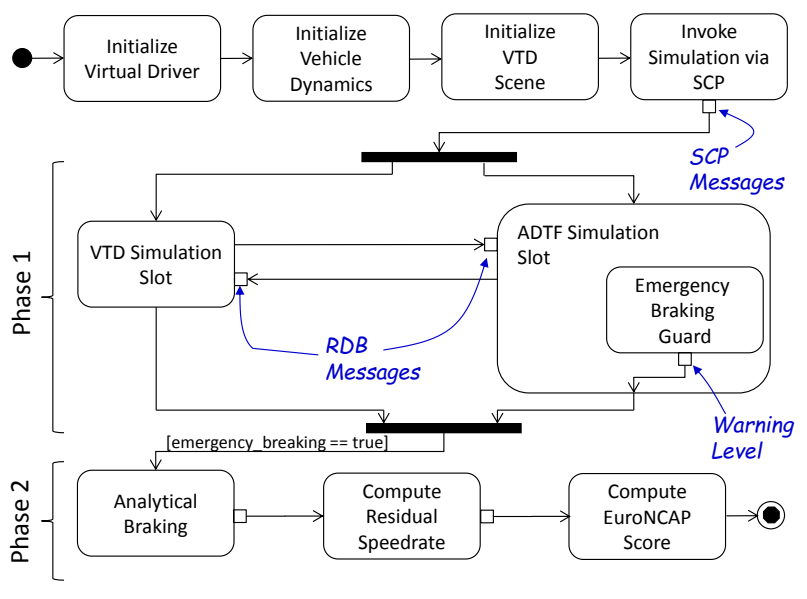

Fig. 2. Workflow of an EuroNCAP Test Simulation

it ends when either the velocity of the VUT is lower than the target's one (i.e. the VUT stops in time) or the it hits the target vehicle. $\left(T_{A E B}\right)$ marks the point of time when the safety function is activated as shown in Fig. 3.

The EuroNCAP test protocol only allows variations between $T_{0}$ and $T_{A E B}$ for the relevant parameters of the VUT. They include lateral deviations up to $0.1 \mathrm{~m}$ from the perfect straight line between both vehicles as well as changes in the VUT's heading angle $\psi$. These variations need to be within certain thresholds so that a given EuroNCAP test is valid.

To systematically investigate the emergency braking guard's behavior in relation to allowed deviations from the ideal trajectory between VUT and the target vehicle, we are considering the simulation's resolution of $0.04 \mathrm{~s}$ as points in time, where we can systematically apply a change to the VUT's parameters influencing its motion model. These variations can be systematically enumerated into a complex graph $\mathcal{G}$. Its root is $T_{\text {end }}$ and all children nodes extend the graph towards $T_{0}$.

Any path $p$ through $\mathcal{G}$ describes a concrete test case that can be used to evaluate the VUT's behavior. To reduce the complexity of $\mathcal{G}$, we limit the paths to only those plausible ones, which are within the allowed tolerance ranges. In this regard, the VUT oscillates around its x-axis with the maximum allowed lateral deviation.

After the function is activated, we are considering that our emergency braking guard needs a delay time of $0.3 \mathrm{~s}$ to fully establish the desired deceleration rate to a limited $3.5 \mathrm{~m} / \mathrm{s}^{2}$ due to illustration purposes.

We are conducting two experimental studies to the aforementioned research questions as described in the following:

Exp-1: Oscillating the VUT alongside the vehicle's $\mathrm{x}$-axis within the allowed EuroNCAP tolerance ranges with the goal to analyze the vehicle's behavior at a test case's boundaries.

Exp-2: Systematically increasing the VUT's lateral deviation from the ideal trajectory with the goal to analyze the vehicle's maximum possible activation range for the safety system.

In the following, the experimental procedure is described to realize the aforementioned experiments.

\section{B. Experimental Procedure}

After an initial initialization, the EuroNCAP test simulation is divided into two phases. The first phase serves for a positioning of the VUT during the simulation run, before an emergency braking action is initiated through the emergency braking guard, cf. Phase 1 in Fig. 2. The first phase begins at the start of the simulation and ends with a warning level output of the emergency braking guard. Afterwards, the second phase is activated whose goal is the computation of the residual velocity and the respective EuroNCAP score, cf. Phase 2 in Fig. 2. In the following, the functional principle of the vehicle dynamics and the virtual driver is described.

The virtual driver processes the particular path $p$ from $\mathcal{G}$ stored in a textual representation and sends commands like accelerate, braking rate, and steering angle as a RDB message to the other modules, e.g. vehicle dynamics and emergency braking guard. The vehicle dynamics, based on the same path $p$, positions the VUT on the prescribed position including 
$(x, y, z)$ and the vehicle heading angle $\psi$ in the simulated scene.

In order to fulfill a simulation run, the VTD scene, virtual driver and vehicle dynamics are initialized according to the scene description and path $p$. Afterwards, the simulation is invoked via the SCP and runs until the warning level of emergency braking guard rises. Based on the distance $D_{x}$ to the target the remaining speed $v_{\text {res }}$ is computed that serves as the input for the EuroNCAP scoring.

All simulations for this paper were executed and evaluated manually. An average time for a simulation test run amounts approximately 4 minutes including subsequent data evaluation. Approximately two hours are required for a CCRs test scenario simulation handling three path variations for example.

\section{Results}

The first subsection shows the results from [Exp-1], the second one the results from [Exp-2], respectively.

1) $\left[\right.$ Exp-1:]: Fig. 4 illustrates varying distances $D_{x}$ to the target vehicles according to the VUT's trajectory with different test speeds at the point in time $T_{A E B}$. According to the related test speed we calculated the TTC, which depends on the deceleration rate $a$ and the distance $D_{x}$ between both vehicles, by using Eq. 1:

$$
\mathrm{TTC}=-\frac{v_{\text {start }}}{a}-\sqrt{\frac{v_{\text {start }}^{2}}{a^{2}}+2 * \frac{D_{x}}{a}}
$$

Each TTC describes the time the VUT needs for travelling $D_{x}$ with a given speed and a constant deceleration. The residual velocity $v_{r e s}$ is estimated by Eq. 2:

$$
v_{r e s}=v_{\text {start }}-a * \mathrm{TTC}
$$

\begin{tabular}{|c|c|c|c|c|c|c|}
\hline \multirow{2}{*}{$\begin{array}{c}\text { test } \\
\text { speed } \\
{[\mathrm{km} / \mathrm{h}]}\end{array}$} & \multicolumn{2}{|c|}{$\begin{array}{c}\text { left-handed } \\
\text { trajectory }\end{array}$} & \multicolumn{2}{c|}{$\begin{array}{c}\text { ideal } \\
\text { trajectory }\end{array}$} & \multicolumn{2}{c|}{$\begin{array}{c}\text { right-handed } \\
\text { trajectory }\end{array}$} \\
\cline { 2 - 7 } & $\begin{array}{c}D_{x} \\
{[\mathrm{~m}]}\end{array}$ & $\begin{array}{c}v_{r e s} \\
{[\mathrm{~m} / \mathrm{s}]}\end{array}$ & $\begin{array}{c}D_{x} \\
{[\mathrm{~m}]}\end{array}$ & $\begin{array}{c}v_{\text {res }} \\
{[\mathrm{m} / \mathrm{s}]}\end{array}$ & $\begin{array}{c}D_{x} \\
{[\mathrm{~m}]}\end{array}$ & $\begin{array}{c}v_{\text {res }} \\
{[\mathrm{m} / \mathrm{s}]}\end{array}$ \\
\hline 10 & 3.97 & 0 & 4.08 & 0 & 3.97 & 0 \\
\hline 15 & 5.46 & 0 & 5.46 & 0 & 5.46 & 0 \\
\hline 20 & 6.98 & 0 & 7.43 & 0 & 6.98 & 0 \\
\hline 25 & 8.72 & 1.33 & 9.28 & 0 & 8.72 & 1.33 \\
\hline 30 & 11.27 & 2.84 & 11.60 & 2.39 & 11.27 & 2.84 \\
\hline 35 & 14.75 & 3.42 & 14.75 & 3.42 & 15.14 & 2.99 \\
\hline 40 & 19.38 & 3.33 & 19.38 & 3.33 & 19.38 & 3.33 \\
\hline 45 & 20.81 & 6.07 & 21.82 & 5.46 & 20.81 & 6.07 \\
\hline 50 & 23.24 & 7.71 & 23.80 & 7.45 & 23.24 & 7.71 \\
\hline
\end{tabular}

Fig. 4. Distances $D_{x}$ and $v_{r e s}$ for each test speed and the left-handed, ideal, and right-handed trajectories.

Fig. 3 illustrates the test case "CCRs AEB $25 \mathrm{~km} / \mathrm{h}$ " with three different trajectories of the VUT towards the target. $T_{0}, T_{A E B}$, and $T_{\text {end }}$ mark the points in time of the official test beginning, trigger point and the test ending. After the emergency braking guard triggered the braking system, the residual velocity $v_{r e s}$ is estimated. Because of the delayed trigger points of the left- and right-handed trajectories, the collision could not be avoided compared to the ideal one.
2) [Exp-2:]: Fig. 5 shows the results of the different distances $D_{x}$ and $v_{\text {res }}$ for an increasing $y$-deviation in rightand left-handed direction, respectively. The calculation of these numbers was done in same way as described for Exp-1. The test speed was set to $50 \mathrm{~km} / \mathrm{h}$. $D_{y}$ represents the lateral position of the VUT when the emergency braking guard was triggered.

\section{Analysis and Discussion}

Now, the results of the two experiments are analyzed and discussed.

1) [Exp-1:]: Fig. 4 reveals a different behavior of the active safety system depending on the lateral position of the VUT. Regularly, a deviation from the ideal path results in a later triggering of the emergency braking guard due to the fact that the algorithm is considering possible evasion trajectories. Except for the $(15 \mathrm{~km} / \mathrm{h})$ and the $(40 \mathrm{~km} / \mathrm{h})$ scenario, each of the test cases shows symmetrical trigger points on the right- and left-handed trajectories wrt. the ideal one. In case of the $(35 \mathrm{~km} / \mathrm{h})$ scenario, the emergency braking guard reveals an unexpected behavior because the left-handed as well as the ideal trajectory result in a higher $v_{r e s}$ than the right-handed one. Regarding the other cases, the trigger points are identically resulting in the same $v_{r e s}$ for the outer trajectories.

\begin{tabular}{|c|c|c|c|c|c|}
\hline \multirow{2}{*}{$\begin{array}{c}\text { test } \\
\text { case } \\
{[\mathrm{m}]}\end{array}$} & \multicolumn{2}{|c|}{$\begin{array}{c}\text { left-handed } \\
\text { trajectory }\end{array}$} & \multicolumn{2}{|c|}{$\begin{array}{c}\text { right-handed } \\
\text { trajectory }\end{array}$} & \multirow{2}{*}{$\begin{array}{c}v_{\text {res }} \\
{[\mathrm{m} / \mathrm{s}]}\end{array}$} \\
\cline { 2 - 5 } & $\begin{array}{c}D_{x} \\
{[\mathrm{~m}]}\end{array}$ & $\begin{array}{c}D_{y} \\
{[\mathrm{~m}]}\end{array}$ & $\begin{array}{c}D_{x} \\
{[\mathrm{~m}]}\end{array}$ & $\begin{array}{c}D_{y} \\
{[\mathrm{~m}]}\end{array}$ & \\
\hline 0.9 & 18.2236 & 0.7846 & 18.2236 & 0.7846 & 8.083 \\
\hline 1.0 & 17.1102 & 0.9158 & 17.1102 & 0.9158 & 8.5516 \\
\hline 1.1 & 17.1114 & 1.0772 & 17.1114 & 1.0772 & 8.5511 \\
\hline 1.2 & 16.5550 & 1.1788 & 16.5550 & 1.1788 & 8.7759 \\
\hline 1.3 & 14.8844 & 1.3018 & 14.8844 & 1.3018 & 9.4186 \\
\hline 1.4 & 14.3280 & 1.4015 & 14.3280 & 1.4015 & 9.6231 \\
\hline 1.5 & 14.3288 & 1.4995 & 14.3288 & 1.4995 & 9.6229 \\
\hline 1.6 & 12.6582 & 1.6202 & 12.6582 & 1.6202 & 10.2124 \\
\hline 1.7 & 12.6588 & 1.6904 & 12.6588 & 1.6904 & 10.2122 \\
\hline 1.8 & 10.9887 & 1.7872 & 10.9887 & 1.7872 & 10.7694 \\
\hline 1.9 & 10.4331 & 1.8803 & 10.4331 & 1.8803 & 10.9485 \\
\hline 2.0 & 7.6487 & 1.983 & 7.6487 & 1.983 & 11.8051 \\
\hline 2.1 & - & 2.0986 & - & 2.0986 & - \\
\hline
\end{tabular}

Fig. 5. Distances $D_{x}$ and $v_{r e s}$ for each test case and the left- and righthanded trajectories, increasing $y$ up to $2.1 \mathrm{~m}$ from the ideal path with a test speed of $50 \mathrm{~km} / \mathrm{h}$.

In addition, the $(25 \mathrm{~km} / \mathrm{h})$-scenario illustrates the possible consequences for a rating later on at EuroNCAP. In case of the ideal trajectory, the collision could be avoided; in the other cases, a collision occurred between both vehicles resulting in a minor evaluation scoring of the whole system.

With these experiments we could show, how a systematic variation of the test parameters within certain tolerance ranges could be modeled for simulation runs, generated as test cases, and an emergency braking guard might be evaluated regarding its trigger points and the resulting $v_{r e s}$. Furthermore, the infrastructure allows a systematic evaluation of an active safety system to reveal unexpected behavior or anomalies. It will enable a better analysis of the system's performance 
and, thus, will support the improvement of the system itself. Moreover, detecting such anomalies enable engineers to more focused test runs in reality validating the results from the simulation runs.

2) [Exp-2:]: The increase of the y-deviation $D_{y}$ outside the officially allowed tolerance range of $0.1 \mathrm{~m}$ reveals that the trigger points will move closer towards the target; and thus the $v_{\text {res }}$ in case of a collision will increase as well.

The difference between the $y$-deviation defining the test case and the measured deviation $D_{y}$ is refereable to the following. The vehicle is oscilliating with an allowed maximum lateral deviation indicated in the test case, but the emergency braking guard was triggerd after the peek. To induce matching values of both test case identifier and $D_{y}$ the frequence of the oscilliation has to be adjusted by further experiments because of a black-box algorithm being used.

Although the lateral deviation is constantly increased, several test cases reveal almost the same $D_{x}$ and $v_{r e s}$ as shown by the $(1.4 \mathrm{~m})$ and $(1.5 \mathrm{~m})$ test cases. Due to the limited possible explanations by the black-box fact further analysis including suppliers is necessary.

Considering the maximum lateral deviation, a braking maneuver will be performed within a range of almost $2.0 \mathrm{~m}$ from the ideal path owing to the fact that a vehicle would safely pass another one outside this range.

Such investigations of an active safety system especially at the system's boundaries and outside the official tolerance ranges will allow a deeper look into its behavior. The attained insights will help to find a robust setting that will meet the consumers' expectations especially in regard to traffic areas. Despite of that, the gained experiences and achieved results by the simulation have be to confirmed in reality by test runs on proving grounds.

\section{E. Threats to Validity}

In the following, threats to validity of our study are discussed according to the guidelines of Runeson and Höst [22].

According construct validity, we are focusing on a structured approach to systematically evaluate an emergency braking guard according to the allowed tolerance ranges in the official EuroNCAP test protocol. Specifically, we selected the maximum allowed deviations from the perfect test setup. Due to the fact that these deviations can occur in reality, their impact needs to be analyzed and evaluated by automotive OEMs to ensure the desired behavior of their vehicles, which is underlining the validity in the construction of our experiments.

Regarding the internal validity of our experiments, we have used a test protocol that was defined by an independent authority to select the deviations in our experiments. Thus, factors that might favor our system under test in the experiments can be ruled out.

Considering external validity, the systematic approach itself is transferable for the evaluation of other instances of AEB/FCW-systems or further EuroNCAP's tests for active safety as well. However, as the system under test is purely evaluated in a virtual environment, selected tests needs to be confirmed by real world experiments on a proving ground to determine the systematic error and to set the context how the findings from the virtual evaluation need to be interpreted.

\section{CONCLUSIONS}

In our work, we presented a simulation-based approach that utilized a graph-based model to systematically evaluate the influence of the tolerance ranges for EuroNCAP tests on the example of an emergency braking guard system. We conducted two experiments to investigate how the lateral deviation from the perfect trajectory affects the time point when the emergency braking guard triggers the braking maneuver. Our first experiment to address our first research question showed that the allowed small variations of $y=$ $\pm 0.1 \mathrm{~m}$ in the official EuroNCAP test cases change the trigger time of the emergency brake maneuver; in some cases of the allowed velocity range of $[10,50] \mathrm{km} / \mathrm{h}$, some anomalies in the behavior could be detected.

Our second experiment was conducted for the $50 \mathrm{~km} / \mathrm{h}$ case to systematically determine the maximum possible lateral deviation up to when the safety system is not activated anymore to investigate our second research question. On the one hand, we could determine the lateral activation range of the system; on the other hand, we could also show that the activation time points move significantly closer towards to target the larger the lateral deviation is.

This simulation-based approach outlined in this work allows the systematic analysis of the software of an active safety system during the development and testing phase for vehicles. Thus, real world experiments can be planned, conducted, and evaluated more effectively because the critical test cases for these experiments are identified systematically.

Our future work will additionally focus on further parameters like a varying velocity of the VUT and the investigation of the moving target in scenarios like "CCRm" and "CCRb". Furthermore, future work needs to be carried out in the models of the system context like the sensor for example that are used in the simulation environment. Thus, further studies to analyze the influence of reduced quality of sensor data can be inspected.

\section{REFERENCES}

[1] T. European New Car Assessment Programme, "Test protocol - aeb systems," EuroNCAP, Test Protocol 1.0, July 2013.

[2] D. Block, S. Heeren, S. Kühnel, A. Leschke, B. Rumpe, and V. Serebro, "Simulations on consumer tests: A perspective for driver assistance systems," in Proceedings of International Workshop on Engineering Simulations for Cyber-Physical Systems, ser. ES4CPS '14. New York, NY, USA: ACM, 2014, pp. 38:38-38:43. [Online]. Available: http://doi.acm.org/10.1145/2559627.2559633

[3] A. Belbachir, J.-C. Smal, J.-M. Blosseville, and D. Gruyer, "Simulation-driven validation of advanced driving-assistance systems," Procedia - Social and Behavioral Sciences, vol. 48, no. 0, pp. 1205 - 1214, 2012, transport Research Arena 2012. [Online]. Available: http://www.sciencedirect.com/science/article/pii/S1877042812028327

[4] F. W. Rauskolb, K. Berger, C. Lipski, M. Magnor, K. Cornelsen, J. Effertz, T. Form, F. Graefe, S. Ohl, W. Schumacher, J.-M. Wille, P. Hecker, T. Nothdurft, M. Doering, K. Homeier, J. Morgenroth, L. Wolf, C. Basarke, C. Berger, T. Gülke, F. Klose, and B. Rumpe, "Caroline: An autonomously driving vehicle for urban environments," in The DARPA Urban Challenge, ser. Springer Tracts in Advanced 
Robotics, M. Buehler, K. Iagnemma, and S. Singh, Eds., vol. 56. Springer Berlin Heidelberg, 2009, pp. 441-508.

[5] C. Berger, Automating Acceptance Tests for Sensor- and Actuator-based Systems on the Example of Autonomous Vehicles. Aachen, Germany: Shaker Verlag, Aachener Informatik-Berichte, Software Engineering Band 6, 2010.

[6] M. Martinus, M. Deicke, and M. Folie, "Virtual test driving hardware-independent integration of series software," ATZelektronik worldwide, vol. 8, no. 5, pp. 16-21, 2013. [Online]. Available: http://dx.doi.org/10.1365/s38314-013-0193-7

[7] K. v. Neumann-Cosel, M. Nentwig, D. Lehmann, J. Speth, and A. Knoll, "Preadjustment of a vision-based lane tracker," in Proceedings on Driving Simulation Conference Europe, 2009, Monaco, 2009.

[8] M. Nentwig, R. Schieber, and M. Miegler, "Hardware-in-the-loop testing of advanced driver assistance systems," ATZelektronik worldwide eMagazine, vol. 6, no. 4, pp. 10-15, 2011. [Online]. Available: http://dx.doi.org/10.1365/s38314-011-0034-5

[9] B. Schick and S. Schmidt, "Evaluation of video-based driver assistance systems with sensor data fusion by using virtual test driving," in Proceedings of the FISITA 2012 World Automotive Congress, ser. Lecture Notes in Electrical Engineering, vol. 196. Springer Berlin Heidelberg, 2013, pp. 1363-1375.

[10] H. Holzmann, K.-M. Hahn, and M. K. B. Klein-Ridder, "Virtual test driving at general motors europe," ATZelektronik worldwide, vol. 3, 2008.

[11] C. Chucholowski, M. Vögel, O. von Stryk, and T.-M. Wolter, "Real time simulation and online control for virtual test drives of cars," in High Performance Scientific and Engineering Computing, 1999.

[12] M. Tideman, "Scenario-based simulation environment for assistance systems," ATZautotechnology, vol. 10, 2010.

[13] M. Tideman, R. Bours, H. Li, T. Schulze, and T. Nakano, "Integrated simulation toolset for ada system development," in Proceedings of the FISITA 2012 World Automotive Congress, ser. Lecture Notes in Electrical Engineering. Springer Berlin Heidelberg, 2013, vol. 200, pp. 25-36. [Online]. Available: http://dx.doi.org/10.1007/978-3-64233838-0_3

[14] W. Hulshof, I. Knight, A. Edwards, M. Avery, and C. Grover, "Autonomous emergency braking test results," in Proceedings of the 23rd International Technical Conference on the Enhanced Safety of Vehicles (ESV), 2013.

[15] EuroNCAP, "Euroncap - the official site of the european new car assessment programme," http://www.euroncap.com/, June 2014.

[16] Audi Electronics Venture GmbH, "Virtual Test Drive - Simulation und Absicherung vorausschauender Funktionen in virtuellem Umfeld," online, Accessed at 03.06.2014. [Online]. Available: http://www.audielectronics-venture.de/aev/brand/de/projekte/virtual_test_drive.html

[17] Vires Simulationstechnologie GmbH, "Virtual Test Drive," online, Accessed at 03.06.2014. [Online]. Available: http://vires.com/products.html

[18] Audi Electronics Venture GmbH, "ADTF Software-Umgebung für Applikationen und Tools," online, Accessed at 03.06.2014. [Online]. Available: http://www.audi-electronicsventure.de/aev/brand/de/leistungen/Entwicklungstools/adtf.html

[19] Elektrobit Corporation, "EB Assist ADTF - Driver Assistance Applications and Safety System Development," online, Accessed at 03.06.2014. [Online]. Available: http://automotive.elektrobit.com/driverassistance/eb-assist-adtf

[20] Oracle, "VirtualBox," Accessed at 10.06.2014. [Online]. Available: https://www.virtualbox.org/

[21] F. Shull, J. Singer, and D. I. K. Sjø berg, Eds., Guide to Advanced Empirical Software Engineering. London: Springer London, 2008. [Online]. Available: http://www.springerlink.com/index/10.1007/978-184800-044-5

[22] P. Runeson and M. Höst, "Guidelines for conducting and reporting case study research in software engineering," Empirical Software Engineering, vol. 14, no. 2, pp. 131-164, Dec. 2008. [Online]. Available: http://link.springer.com/10.1007/s10664-008-9102-8 